\title{
Ambiente, comunidad y salud: un trabajo de campo sobre la leptospirosis en San José del Rincón, Argentina
}

\author{
Valeria Yelena Kuttel \\ ykuttel@unl.edu.ar \\ (iD) orcid.org/0000-0002-6857-740X

\section{Luz María Rodeles} \\ Centro de Estudios en Salud Global \\ de la Facultad de Ciencias Médicas \\ luzrodeles@gmail.com \\ (iD) orcid.org/0000-0003-0793-4810
}

\author{
Larisa Ivón Carrera \\ carreralarisa@gmail.com \\ (iD) orcid.org/0000-0002-8157-1301

\section{Verónica Reus} \\ vreus@fcm.unl.edu.ar \\ (iD) orcid.org/0000-0003-2271-5675
}

Universidad Nacional del Litoral, Argentina.
Ambiente y extensión universitaria /

Desafíos de gestión
RECEPCIÓN: 30/06/21

ACEPTACIÓN FINAL: 03/10/21

\section{Resumen}

En el artículo se propone reflexionar sobre la incidencia de las intervenciones universitarias en la salud y el bienestar de la población a partir de un trabajo vinculado a la enfermedad de la leptospirosis. En particular, frente al desarrollo de factores ambientales, sociales y económicos que pueden propiciar brotes epidemiológicos. A través de la experiencia llevada adelante por la Facultad de Ciencias Médicas de la Universidad Nacional del Litoral, la Municipalidad de San José del Rincón y otros actores sociales de la localidad en diferentes barrios de la ciudad, se evidencia el impacto de las intervenciones intersectoriales con la finalidad de reducir inequidades y mitigar los problemas de salud.

Por último, se da cuenta de la importancia de la incorporación de nuevos dispositivos, tales como la georreferenciación, para la promoción de la salud y la construcción de políticas preventivas.

Palabras clave:salud ambiental; trabajo intersectorial; determinantes de salud; extensión universitaria; leptospirosis.
Environment, community and health: a field work on leptospirosis in San José del Rincón, Argentina

\section{Abstract}

In the following article, we propose to reflect on the incidence of university interventions on the health and well-being of the population based on a study related to the disease of leptospirosis. In particular, when faced with the development of particular environmental, social and economic factors, which can lead to epidemiological outbreaks.

Through the experience carried out by the Faculty of Medical Sciences of the National University of the Litoral, the Municipality of San José del Rincón and other social actors of the locality, in different neighborhoods of the city, it is evident the impact of intersectoral interventions, in order to reduce inequities and mitigate health problems.

Finally, it realizes the importance of the incorporation of new devices, such as georeferencing for health promotion and the construction of preventive policies.

Keywords: enviromental health; intersectoral work; health determinants; college extension; leptospirosis.

\author{
Meio ambiente, comunidade e saúde: \\ um trabalho de campo sobre a leptospirose \\ em San José del Rincón, Argentina
}

\section{Resumo}

O artigo a seguir se propõe refletir sobre a incidência de intervenções universitárias sobre a saúde e o bem-estar da população a partir de um estudo relacionado à doença da leptospirose. Em particular, quanto ao desenvolvimento de fatores ambientais, sociais e econômicos particulares, que podem levar a surtos epidemiológicos. Através da experiência realizada pela Faculdade de Ciências Médicas da Universidade Nacional del Litoral, a Câmara Municipal de San José del Rincón e outros atores sociais da cidade, em diferentes bairros da cidade, é evidente o impacto das intervenções intersetoriais, a fim de reduzir as iniquidades e mitigar os problemas de saúde. Por fim, percebe-se a importância da incorporação de novos dispositivos, como o georreferenciamento para a promoção da saúde e a construção de políticas preventivas.

Palavras-chave: saúde ambiental; trabalho intersetorial; determinantes da saúde; leptospirose.

Para citación de este artículo: Kuttel, Y.; Rodeles, L. M.; Carrera, L. I. y Reus, V. (2021). Ambiente, comunidad y salud: un trabajo de campo sobre la leptospirosis en San José del Rincón, Argentina. +E: Revista de Extensión Universitaria, 11(15), e0015. doi: 10.14409/extension.2021.14.Jul-Dic.e0015 


\section{Introducción}

La Facultad de Ciencias Médicas (FCM) de la Universidad Nacional del Litoral (UNL) viene participando activamente con diversos proyectos en la zona de la costa del litoral santafesino, aledaña al corredor de la Ruta Provincial 1, que incluye la comunidad de San José del Rincón (Kuttel, Birri y Reus, 2016; Blesio, 2020). Esta continuidad en el territorio posibilitó vincularse en forma colaborativa y sinérgica con la Municipalidad, el Sistema para la Atención Médica de la Comunidad (SAMCo), ${ }^{1}$ las escuelas y organizaciones de la sociedad civil de la región. Permitió a la universidad, y en este caso de la facultad, cumplir con su misión social de contribuir a la mejora de algunos aspectos vinculados a problemas sociales y de salud. Asimismo, construir en el territorio con su red de actores sociales un escenario favorable para fortalecer las actividades de docencia, investigación y extensión.

En este recorrido se articularon prácticas curriculares en territorio, proyectos de extensión y de investigación orientados a problemas sociales y productivos. Los estudiantes y docentes abordaron en ese contexto de aprendizaje los contenidos de los planes de estudio en situaciones reales, con sus problemas y actores, y produjeron conocimiento con un equipo interdisciplinario e intersectorial. Así, las aulas se transformaron en plazas, calles, patios de escuelas, vecinales en donde se aprende con otro, es decir, no solo con sus compañeros de cursada o con su docente, sino también con el presidente de la vecinal, la maestra de la escuela, los niños, la vecina, etc., para juntos trabajar en acciones de salud.

En esa línea, y siguiendo el objetivo número 3 de los Objetivos de Desarrollo Sostenible de Naciones Unidas, "Garantizar una vida sana y promover el bienestar para todos en todas las edades", se plantea una reflexión sobre los problemas de gestión ambiental y su impacto en la salud a partir del análisis de un caso concreto.

Este artículo presenta una contextualización de la situación socioambiental en San José del Rincón en la que se explica su impacto en la salud y, respecto de esta, se atiende a sus determinantes en las personas. También se comparte el detalle de las intervenciones en el territorio, en donde se hace foco en el accionar de cada uno de los integrantes del equipo. Además, se incluyen los resultados del relevamiento obtenido en Acería, Centro, Cina Cina, Los Espinillos y Villa Añati. Finalmente, se apuntan algunas reflexiones sobre los resultados alcanzados.

\section{La leptospirosis como problema ambiental en el litoral argentino}

El ambiente es el medio en donde las personas y las comunidades nacen, crecen y se desarrollan. El mismo los protege y les brinda las posibilidades para obtener alimento, pero en ocasiones, puede constituirse en un espacio de riesgo para la vida y, consecuentemente, para la salud individual y colectiva.

Para que esto no suceda, es importante mantener buenas condiciones de calidad de aire y agua. Asimismo, disminuir o evitar la exposición a agentes químicos y biológicos, radiaciones y ruidos ambientales, trabajar en prácticas agrícolas sustentables, mejorar los entornos urbanizados precarizados y mitigar el impacto del cambio climático para modificar la situación actual, puesto que, según la Organización Mundial de la Salud (OMS), el 23 \% de las 
muertes está relacionado con causas medioambientales, lo cual incide en mayor grado en las personas y poblaciones más vulnerables, como los niños y adultos mayores en situación de pobreza e indigencia (Télam, 2021).

Es imprescindible reducir y controlar los problemas ambientales para mejorar la calidad de vida de nuestras comunidades.

Dentro de los riesgos ambientales, nos encontramos con la exposición a agentes biológicos, como insectos y animales que transmiten enfermedades. Entre estas se encuentran las enfermedades zoonóticas, como, por ejemplo, la leptospirosis, que es una afección bacteriana de distribución mundial que aparece en forma aislada o en brotes epidémicos estacionales, constituye un problema emergente de la salud pública, y cuya mortalidad es cercana al $10 \%$. El hombre se infecta ocasionalmente y sufre de una enfermedad sistémica, febril y aguda, causada por espiroquetas del género Leptospira especie interrogans (Vinetz, 2001). Suelen transmitirse al hombre por intermedio del agua, los alimentos o el terreno húmedo contaminados por la orina de animales infectados. Las leptospiras ingresan a través de la piel erosionada o de las mucosas orofaríngea, nasal, ocular, aunque también pueden penetrar por la piel íntegra si permanece inmersa en agua por un tiempo.

Numerosos factores ambientales, sociales y económicos, son determinantes en la presentación de casos y brotes epidémicos de leptospirosis. Estos últimos son más frecuentes durante desastres naturales, principalmente inundaciones o períodos de lluvias intensas. La urbanización descontrolada con deficiente saneamiento ambiental, la presencia de basurales y la proliferación de roedores componen el ambiente ideal para la aparición de casos. Si a esto le sumamos la presencia de animales de producción y domésticos sin control sanitario, tendremos el escenario propicio para un grave problema de salud pública (Ministerio de Salud de Argentina, 2014). Varios de estos aspectos mencionados se encuentran presentes en el municipio de San José del Rincón (Santa Fe, Argentina), considerando que está a la orilla del río San Javier y cuenta con zonas rurales. Es una localidad con aproximadamente 11000 habitantes que ha sufrido en las últimas décadas un aumento poblacional debido a que personas que vivían en la capital santafesina decidieron radicarse en esta ciudad que dista apenas 7 kilómetros de aquella, lo que genera un gran impacto ambiental y de demanda de servicios públicos. La localidad cuenta con un SAMCo y una incipiente red de agua potable; la mayoría de las casas posee agua de pozo y para consumo humano se compra agua envasada.

Actualmente, la leptospirosis se considera un problema de salud pública en todo el territorio argentino (y las provincias de Santa Fe, Buenos Aires y Entre Ríos se registran dentro de las zonas endémicas (Ricardo, 2018). San José del Rincón no escapa a esta realidad, su situación geográfica y el predominio de zonas rurales favorecen la alta tasa de leptospirosis. En este contexto, la mejor forma de controlar las enfermedades es prevenirla por medio de actividades de promoción de la salud, saneamiento básico, protección de grupos de riesgo y de animales domésticos.

\section{La gestión de la salud ambiental: los aportes de la universidad}

Los determinantes del estado de salud abarcan desde factores ambientales, biológicos, conductuales, sociales, económicos, laborales, culturales y los servicios sanitarios como respuesta organizada y especializada de la sociedad para prevenir la enfermedad y restaurar la salud. 
El desarrollo de la salud ambiental implica, entre otras cosas, el énfasis en la responsabilidad colectiva sobre la salud y el papel del Estado en la promoción y protección de la salud; el enfoque poblacional el compromiso y trabajo asociado con la población a la que sirve (Garza-Almanza, 1997).

Cuellar (2008) nos plantea un marco conceptual para la salud ambiental urbana que nos permite observar cómo la salud y la calidad de vida, tanto individuales como colectivas, están intersectadas e interrelacionadas por cuatro aspectos principales: la gestión de la contaminación urbana, las áreas verdes como medidas de remediación, la vivienda saludable, y los servicios públicos adecuados. Cada uno de ellos tiene que ver con cuestiones colaterales, como la contaminación, la demanda de servicios y la participación social permanente, elemento básico en la consecución de un estado de vida saludable.

Desde este modelo, uno de los puntos relevantes a considerar para la protección del ambiente y de la vida es lograr la reducción y control de los factores ambientales, más precisamente de los peligros ambientales tradicionales y modernos, ambos asociados a distintas facetas del desarrollo social y económico. Su efecto sobre la salud variará de tipo, intensidad y magnitud según la clase de peligro, el nivel de exposición y el número de afectados. Las enfermedades más importantes suelen asociarse a más de un tipo de exposición, y para causarlas los peligros actúan junto con los factores genéticos, la nutrición, los riesgos del estilo de vida, entre otros (PAHO, 2010). Una buena gestión ambiental minimiza los peligros con medidas de prevención efectivas y económicas.

En este marco, la FCM-UNL se encuentra comprometida con su medio y desarrolla diversas acciones surgidas de un diálogo permanente con diferentes actores gubernamentales provinciales en sus distintas dependencias, locales, como municipios y comunas, y con organizaciones de la sociedad civil. Estos encuentros se constituyen en mesas de trabajo que definen agendas de temas/problemas en investigación y extensión. Entre ellas, las principales líneas de investigación de la Facultad son: diabetes e hipertensión, factores de riesgo, Chagas, nutrición, tecnología en educación, tecnología móvil en salud, medicina forense, medicina laboral y legal, cáncer genito mamario, educación médica, plaguicidas y COVID-19. En cuanto a las principales acciones de extensión, se destacan: parto respetado, abordaje sobre el dengue, toxoplasmosis, síndrome urémico hemolítico, cáncer de colon, envejecimiento activo, salud rural, lactancia materna, hábitos saludables, salud visual, cultura y salud.

Cabe mencionar que, desde la FCM, pensando en la formación de profesionales socialmente responsables y comprometidos, la misma carrera de Medicina:

"se sustenta en el Aprendizaje Basado en Problemas (ABP) del medio social como estrategia pedagógica que contribuye a la formación integral de los futuros profesionales desde el razonamiento y el juicio crítico. Esta metodología de enseñanza se implementa durante todo el transcurso de la carrera desde las diversas áreas del plan de estudio que involucran distintas disciplinas. Así, al estudiante se lo implica como protagonista y creador de sus propios aprendizajes al poder desarrollar competencias para el abordaje de temas-problemas relacionados con la salud. Por su parte, el docente actúa como un tutor que ofrece el andamiaje para sostener el autoaprendizaje y la educación continua en diferentes escenarios (centros de salud, centros de cuidado infantil, centros de acción familiar, escuelas, y ONG), para sostener y mejorar la salud pública”. (Kuttel, Birri y Reus, 2016) 
A partir de la lógica del trabajo intersectorial y comunitario, se comprende cómo abordar los determinantes sociales de la salud que, como es sabido, se encuentran mayormente fuera del sector Salud. Por lo que resulta fundamental en estas instancias la participación de gestores institucionales en salud pública que puedan traducir las demandas, problemáticas y necesidades de los ciudadanos, en acciones de salud para mejorar las condiciones de vida. Desde estos procesos de gobernanza se garantiza el derecho de la ciudadanía a participar para contribuir a mejorar las políticas públicas (Kuttel, Birri, Blesio y Kuttel, 2017).

En este sentido, los trabajos de investigación y extensión son espacios que generan información e instrumentos confiables para la toma de decisiones y, precisamente, para el fortalecimiento de las políticas públicas en salud.

\section{La experiencia en el territorio}

En 2014, la FCM, la Municipalidad de San José del Rincón y el SAMCo local iniciaron Prácticas de Extensión de Educación Experiencial ${ }^{2}$ (PEEE) y voluntariado universitario. Esto permitió ir logrando una dinámica de trabajo en red entre las instituciones y el desarrollo de acciones de salud a través de la participación de estudiantes de medicina, obstetricia y enfermería. Sobre esa base, en 2017 se pudo formular y llevar a cabo un proyecto de investigación orientado a problemas sociales y productivos (CAl+D).

Dentro de los propósitos de ese CAI+D, se destaca el aporte respecto de la disminución de la incidencia de leptospirosis con la incorporación de nuevas tecnologías. Se desarrolló un mapa de densidad epidemiológica de zonas de riesgo de contagio para poder referenciar con casos detectados probables y confirmados.

Este proyecto posibilitó que estudiantes de la práctica final obligatoria de la carrera de Medicina pudieran llevar a cabo, en su rotación en el primer nivel de atención, actividades en territorio, entre las que se pueden mencionar acciones de sensibilización en la comunidad, una identificación de los determinantes ambientales que más influyen sobre la incidencia de leptospirosis en San José del Rincón, y establecer relaciones con los focos epidemiológicos hallados mediante georreferenciación.

Esto se realizó en cinco barrios del municipio: Acería, Centro, Cina Cina, Los Espinillos y Villa Añatí (ver Figura 1).

Se hicieron encuestas en forma censal sobre factores de riesgo individuales y ambientales de leptospirosis y un relevamiento ambiental que implicó, a partir de análisis cartográficos en cada barrio dividido en conglomerados recorridos en transectas lineales, registrar con referentes locales, indicadores que se consideran factores de riesgo ambientales para leptospirosis: receptáculos de agua dulce, cestos en altura, residuos domésticos en vía pública, falta de recolección de residuos, minibasurales/basurales a cielo abierto, escombros, restos de poda, pastos sin cortar, galpones, estructuras abandonadas, chatarrería, perros domésticos, perros callejeros, gatos, caballos, gallinas, vacas, cerdos, materia fecal de animales domésticos, zona pesquera, zona inundable por lluvias, zona inundable por crecida.

2) Las prácticas de extensión se desarrollan en el marco de las propuestas curriculares de las carreras de la UNL con el propósito de profundizar alternativas de formación de profesionales sólidos y fundamentalmente comprometidos con la sociedad (Kuttel, Birri y Reus, 2016). 
Figura 1. Ubicación georreferenciada de los barrios relevados en San José del Rincón

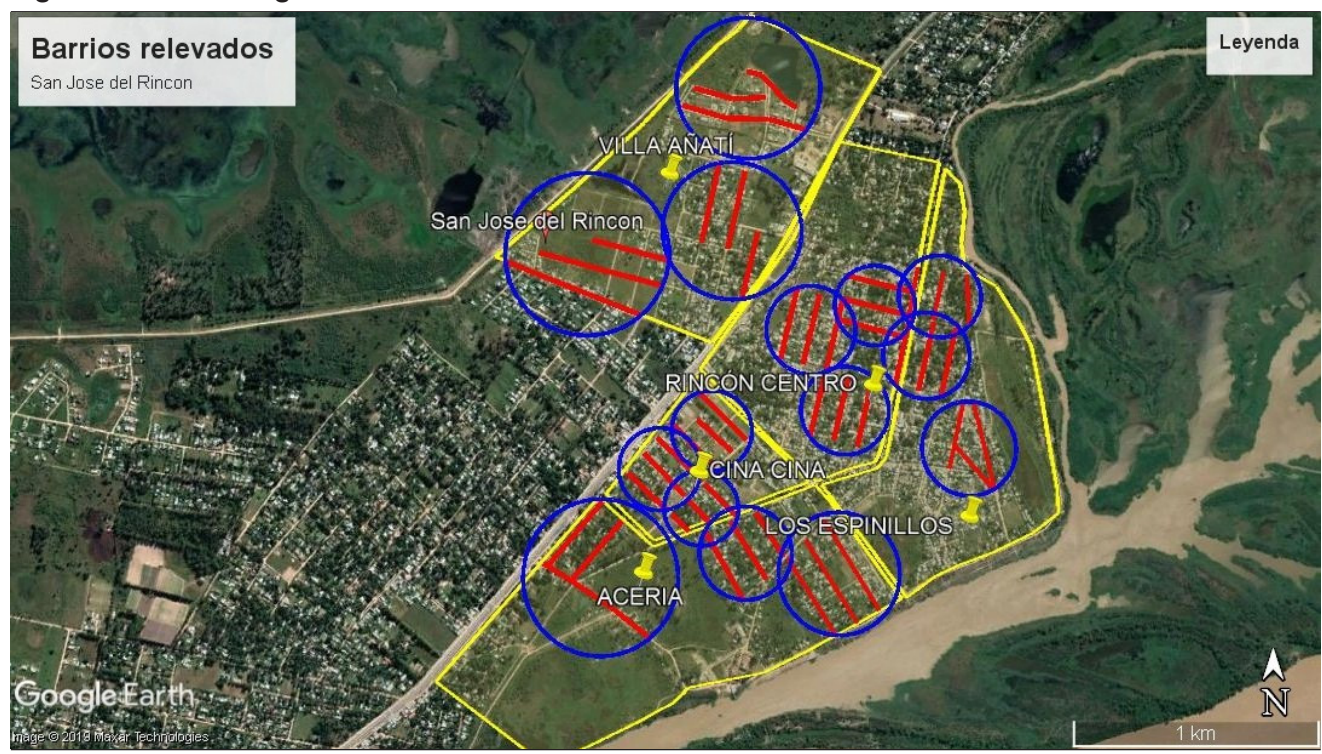

Fuente: Google Earth.

La planificación de la actividad implicó una coordinación con el equipo docente, los referentes del área de salud del municipio y trabajadores de salud del SAMCo.

Además, se desarrollaron capacitaciones para los estudiantes involucrados sobre las estrategias de relevamiento territoriales, los tópicos a relevar y herramientas de comunicación asertiva para entrar en diálogo con las personas de la comunidad.

Las llegadas a territorio por parte de los equipos universitarios (docentes y estudiantes) fueron anticipadas mediante diversas estrategias de comunicación por los medios locales de televisión, radio y redes sociales con que cuenta el municipio, lo que permitió lograr un mayor éxito, ya que los vecinos estaban informados acerca de la actividad que iban a llevar adelante los equipos universitarios junto con los referentes del área de salud del municipio.

Estas actividades fueron acompañadas por acciones de sensibilización por parte de los estudiantes sobre la importancia de realizar acciones de prevención de la leptospirosis aprovechando la oportunidad como escenario de educación para la salud.

En el desarrollo del proyecto se constituyó un equipo compuesto por referentes universitarios, municipales y trabajadores de la salud, quienes planificaron, diseñaron e implementaron las actividades. La información relevada fue sistematizada y analizada por los estudiantes en colaboración con los docentes.

Una vez integrada la información del relevamiento ambiental y georreferenciada, se construyó la Figura 2 a los fines de reflejar los datos. 
Figura 2. Relevamiento ambiental en San José del Rincón

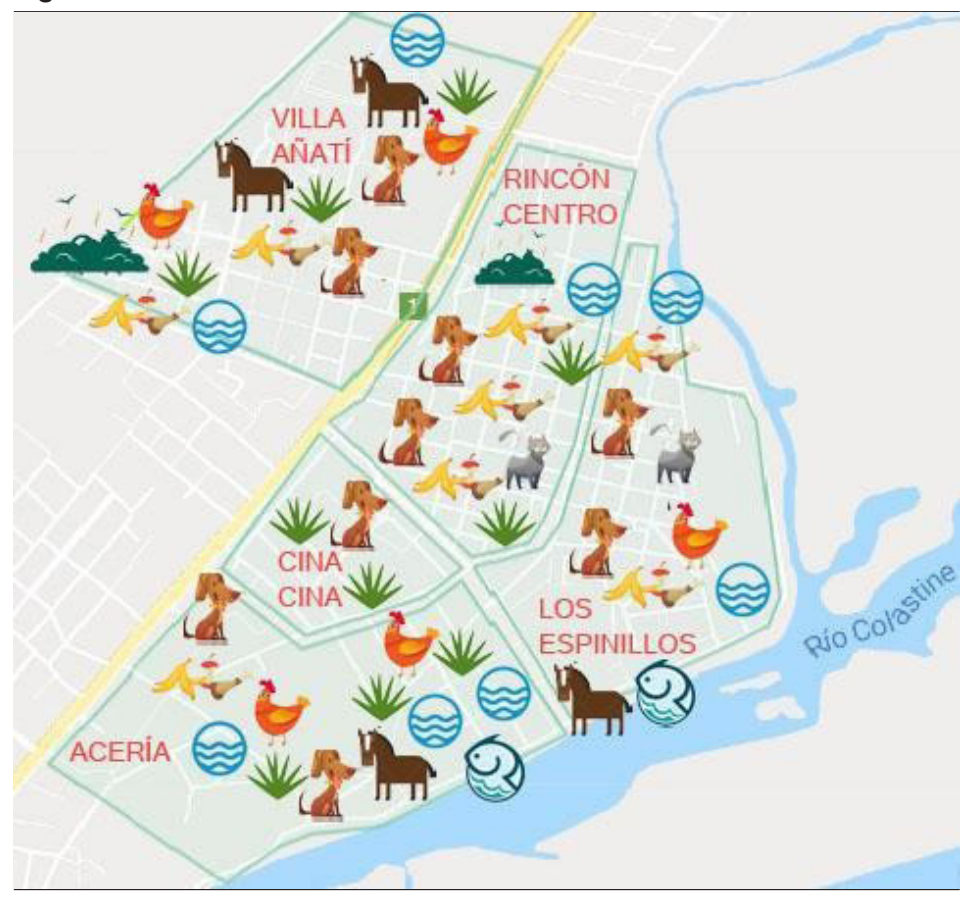

Fuente: elaboración del equipo del Proyecto.

Del total de encuestas $(n=767)$ efectuadas a los vecinos de los diferentes barrios de la ciudad de San José del Rincón se extrajeron los siguientes datos como relevantes y necesarios de ser tenidos en cuenta.

- En cuanto a exposición ocupacional: el $56 \%$ ( $n=431)$ refirió realizar trabajos considerados de bajo riesgo, mientras el $43 \%(n=336)$ restante desarrolla actividades vinculadas a una mayor exposición, como trabajo en comercios con depósitos de alimentos un $20 \%$ ( $n=149$ ), jardinería el $11 \%(n=86)$ y albañilería un $10 \%(n=73)$. Fueron minoritarios $(\leq 3 \%)$ el trabajo agrícola, la recolección de residuos y alcantarillado. Pese a ser un distrito costero, solo el 5 $\%(n=39)$ dijo realizar actividades relacionadas con la pesca.

- Según lo relevado, la mayoría utiliza calzado para trabajar (95\%); sin embargo, un $2,7 \%(n=24)$ no utiliza calzado cerrado, siendo un 7,5 \% $(n=39)$ los que no se protegen en este sentido.

- Un $56 \%(n=429)$ de la población refiere haber visto roedores en la zona, en su mayoría en espacios públicos, en la calle (46\%; $\mathrm{n}=281)$ y en el peridomicilio $(40 \% ; n=241)$.

En la Tabla 1 se resumen los datos de las encuestas realizadas a los individuos en los diferentes barrios sobre los aspectos relacionados con la presencia de receptáculos de agua dulce, residuos en vía pública y presencia de animales. 
Tabla 1. Manejo de residuos, disponibilidad de agua dulce y presencia de animales en San José del Rincón

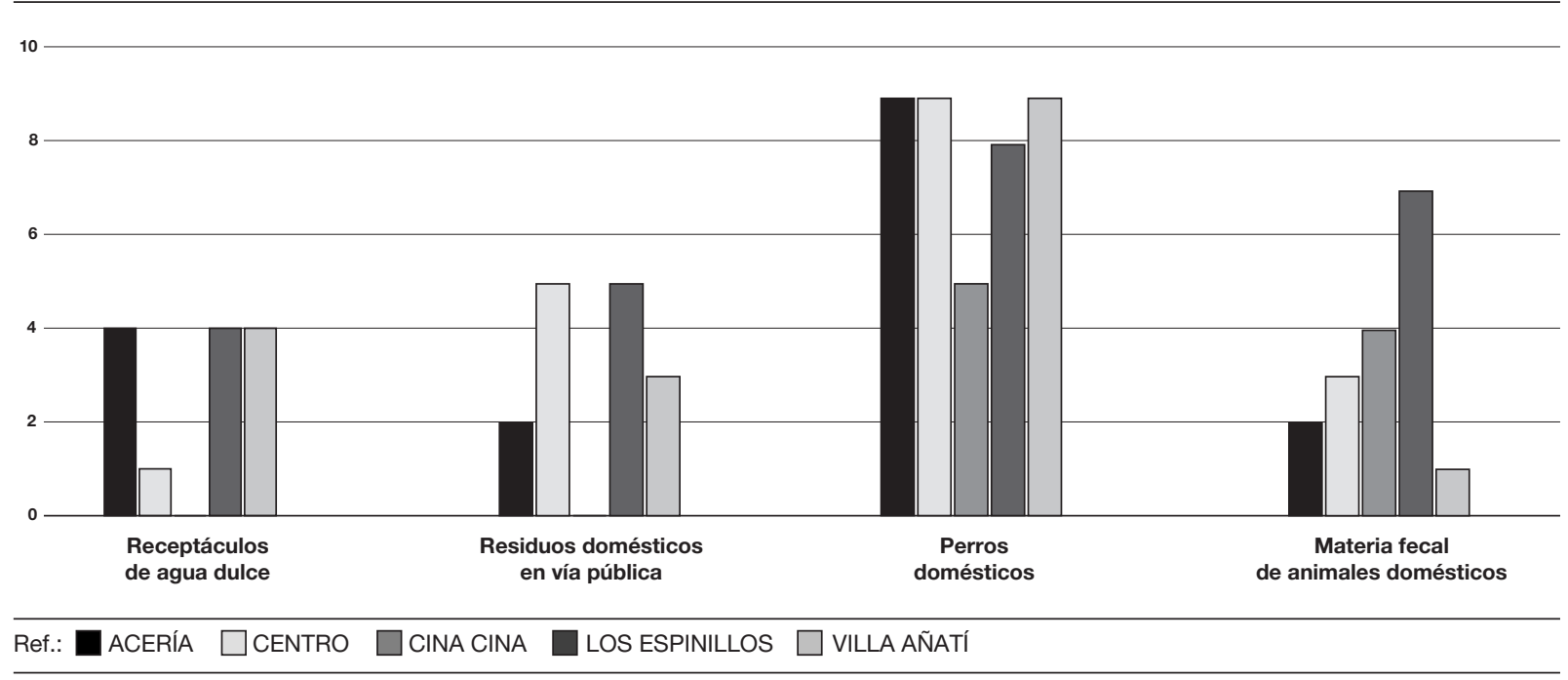

Fuente: elaborado por el equipo del proyecto

A partir de los datos relevados en el campo y considerando los generados en las encuestas, se considera que los barrios que presentan condiciones de mayor riesgo para la transmisión de leptospirosis son Villa Añatí y Los Espinillos.

Y atento a la información aportada por la población, se trabajó en la construcción de un mapa de riesgo que conjugara esta información y se integró al diseño de intervenciones a diferentes niveles, incluyendo acciones de saneamiento ambiental jerarquizadas según los datos obtenidos de cada barrio con los actores sociales del municipio.

Los estudiantes llevaron adelante trabajos de sensibilización sobre la temática en los barrios antes mencionados.

\section{Algunas consideraciones}

Los estudiantes pudieron resignificar conceptos y prácticas que fueron aprendiendo a lo largo de su carrera de Medicina en una situación de contexto real, en una comunidad en particular. Así, conceptos de salud ambiental, salud comunitaria, Atención Primaria de la Salud, epidemiología, zoonosis, historia clínica familiar, educación para la salud y estadística pudieron ponerse en juego en esta práctica en particular.

Estas experiencias de extensión se valoran por intermedio de informes individuales en donde relatan la actividad desarrollada, reflexionan sobre qué aprendieron y cómo valoran la experiencia a partir de: la posibilidad de seguir construyendo a partir de conocimientos previos, la adaptación a nuevas situaciones, la capacidad de organización del trabajo, gestión de la información, registro de datos y de ejecución de tareas planificadas. Además, evalúan la capacidad de escucha frente a las inquietudes expresadas por otros actores, actitud para observación y posterior análisis, capacidad para reconsiderar posiciones propias en el diálogo con otros actores y sus pares durante el desarrollo de las actividades, adaptación al territorio, capacidad para tomar decisiones y asumir el trabajo colaborativo. Este informe es 
tomado por los docentes para planificar las instancias grupales, nominadas "talleres de reflexión", en donde se recupera el accionar grupal y la participación en un problema concreto. Se resignifican los temas-problema abordados en la práctica extensionista.

Otro aspecto a tener en cuenta como indicador a la contribución de la experiencia en la formación del estudiante son las publicaciones que ellos producen en congresos y encuentros de jóvenes investigadores. Esta actividad en particular fue publicada por estudiantes en un Congreso del Foro de Facultades y Escuelas de Medicina Pública y un Encuentro de Jóvenes Investigadores.

\section{Conclusiones}

La propuesta permitió consolidar el vínculo con las instituciones de salud y el gobierno local. El SAMCo dispone de información que posibilita a los profesionales de la salud identificar los lugares con mayor riesgo socioambiental a tener en cuenta ante los posibles diagnósticos de enfermedades vinculadas con determinantes ambientales, más precisamente de leptospirosis.

Además, favoreció el fortalecimiento intersectorial con el gobierno local y la Universidad en estrategias de promoción de la salud y prevención de enfermedades. Ello a través de la instalación de un instrumento de georreferenciación, que es clave para la continuidad en la promoción de la salud y en la construcción de políticas preventivas.

En suma, el municipio cuenta con una herramienta para controlar y mitigar problemas ambientales que afectan a la salud de las personas, posee información sistematizada y georreferenciada para la toma de decisiones en la gestión ambiental de su comunidad, de barrios en particular, y del diseño de un dispositivo para poder aplicarlo en los barrios restantes.

La Universidad reforzó su compromiso social, aportó información, recursos y tecnologías para la resolución de los problemas ambientales.

Asimismo, se favoreció el intercambio de saberes y sentidos sobre leptospirosis y su relación con la salud ambiental en la comunidad, lo que resultó enriquecedor tanto para actores sociales como para estudiantes y docentes que exploraron diferentes dimensiones del problema, más allá de los datos.

Por último, este proyecto constituyó un espacio para que los estudiantes desarrollen en su último año de la carrera una Práctica de Educación Experiencial, en la cual trabajaron en un mapeo de determinantes socioambientales sobre un problema de salud en particular, la leptospirosis, y referenciaron así una problemática de una comunidad con una estrategia de un efector de salud intersectorialmente con una gestión local. Estos puntos son ejes para la formación de un médico capaz de prestar atención integral e integrada en diferentes aspectos de la salud individual, colectiva y medioambiental mediante acciones de promoción, prevención de las enfermedades, diagnóstico, tratamiento y recuperación de la salud en el marco de la Atención Primaria de la Salud.

\section{Referencias bibliográficas}

Acevedo, G.; Martínez, D. y Utz, L. (s.f.). Manual de Medicina Preventiva y Social I. Unidad La salud y sus determinantes. La salud pública y la medicina preventiva. http://preventivaysocial.webs.fcm.unc.edu.ar/files/2014/04/MPyS-1-Unidad-1-Determinantes-de-la-Salud-V-2013.pdf 
Blesio, H. (2020). Mesa de Trabajo Intersectorial en Salud de Santa Fe: una estrategia de participación colectiva para la prevención del dengue. +E: Revista De Extensión Universitaria, 10(12). https://doi.org/10.14409/ extension.2020.12.Ene-Jun.9090

Cuellar, H. (2008). Conceptualización de la salud ambiental: teoría y práctica (parte 1). Revista Peruana de Medicina Experimental y Salud Pública, 25(4), 403-409. http://www.scielo.org.pe/scielo.php?script=sci_arttext\&pid=S1726-46342008000400010\&lng=es\&tIng=es

Garza-Almanza, V. (1997). Salud y ambiente en el desarrollo sostenible. Ambiente sin Fronteras, 1(6), 6-11.

Kuttel, Y.; Birri, M. y Reus, V. (2016). Los dispositivos para integrar la docencia con la extensión en la Facultad de Ciencias Médicas de la Universidad Nacional del Litoral. +E: Revista de Extensión Universitaria, 6(6), 188-193. https://doi.org/10.14409/extension.v1i6.6329

Kuttel, Y.; Birri, M; Blesio, H; Kuttel, I. (2017). Construir salud entre todos. Desafío de la extensión universitaria. En Menéndez, G.; Urbani, M. e lucci, C. (Comps.) (2017). III Congreso de Extensión de la Asociación de Universidades del Grupo Montevideo. Relatos de experiencias de extensión. UNL.

Lalonde, M. (1974). A new perspective on the health of Canadians, a working document. Minister of National Health and Welfare. https://fundadeps.org/recursos/Informe-Lalonde/

Ministerio de Salud de Argentina (2014). Enfermedades infecciosas leptospirosis, diagnóstico de Leptospirosis. Guía para el equipo de Salud. https://www.rosario.gob.ar/mr/epidemiologia/vigilancia/vigilancia-intensificada/leptospirosis-1/leptospirosis-guia-para-el-equipo-de-salud/view

ONU (Organización de las Naciones Unidas) (s.f.). Objetivos de Desarrollo. https://onu.org.gt/objetivos-de-desarrollo/

OPS (Organización Panamericana de la Salud) (2010). Ambiente y salud. https://www. paho.org/hq/dmdocuments/2010/Sanemiento-Capitulo1.pdf

Ricardo, T. (2018). Evaluación del riesgo de leptospirosis en asentamientos marginales ribereños de Santa Fe, Argentina, mediante un enfoque "Una Salud". [Tesis de Doctorado en Ciencias Veterinarias]. Dpto. de Ciencias Naturales, Facultad de Humanidades y Ciencias. UNL.

Télam (2021, abril 7). Informe de la OMS. "Casi una de cada cuatro muertes en el mundo se debe a causas medioambientales". https://www.telam.com.ar/notas/202104/549980-muerte-medioambiente.html

Vinetz, J.M. (2001) Leptospirosis. Curr. Opin. Infec.t Dis. 2001 14(5), 527-538. 10.1097/00001432-20011000000005. PMID: 11964872. 